\title{
GENERALIZATIONS OF WEAKLY COMPACT OPERATORS $\left(^{1}\right)$
}

\author{
BY \\ RICHARD H. HERMAN
}

1. Introduction. This paper is concerned with developing generalizing concepts of weakly compact operators analogous to the generalization of compact operators by strictly singular operators. The latter class is given by:

1 Definition. $T \in[X, Y]\left({ }^{2}\right)$ is said to be strictly singular if whenever $T$ has a bounded inverse on $M$, a closed subspace of $X, M$ is finite dimensional [6].

We introduce two definitions below which give rise to distinct classes of operators containing properly (in general) the class of weakly compact operators. The discussion includes conditions for these operators to fall into more familiar classes and the effect of conjugation on their properties.

2 Definition. Let $T \in[X, Y]$. $T$ is said to be almost weakly compact (a.w.c.) if, whenever $T$ has a bounded inverse on a closed subspace, $M$, then $M$ is reflexive.

It is clear that the class of almost weakly compact operators includes both the strictly singular and the weakly compact operators.

We now give an example to show that these classes are distinct.

3 ExAmple. Let $X=l_{2} \times l_{1}$ and $Y=l_{2} \times c_{0} . U=I \times T: X \rightarrow Y$ where $I$ is the identity map and $T\left(\left(\alpha_{i}\right)\right)=\left(\left(\sum_{n=i}^{\infty} \alpha_{n}\right)\right)$. Clearly $U$ is not strictly singular, and, since the image of the unit vectors in $l_{1}$, under $T$, has no weakly convergent subsequence, it follows that $U$ is not weakly compact. To prove $U$ is a.w.c. it suffices, since $l_{2} \times l_{1}$ is weakly sequentially complete, to show that every nonreflexive subspace of $l_{2} \times c_{0}$ contains a copy of $c_{0}$, which is not weakly sequentially complete [1]. To this end, let $M$ be one such subspace. We define $c^{n}$ to be those elements belonging to $c$ which have zeros after the $n$th coordinate. Since $M$ is not reflexive, and $l_{2} \times c^{n}$ for all $n$ is, it follows that for no $n$ does the projection $P_{n}: M \rightarrow l_{2} \times c^{n}$ have a bounded inverse. Hence, there exists in $M$ a sequence $\left\{m_{n}\right\}$ such that $\left\|m_{n}\right\|=1$ and

$$
\left\|P_{n} m_{n}\right\|<\frac{1}{2^{n}}
$$

Let the norm in $l_{2} \times c_{0}$ be such that if $m=(x, y)$, then $\|m\|=\|x\|_{l_{2}}+\|y\|_{c_{0}}$ we consider the following subsequence:

Received by the editors February 20, 1967.

(1) This paper is taken from Chapter I of the author's doctoral dissertation and was done while the author was a NDEA Fellow at the University of Maryland.

$\left.{ }^{2}\right)[X, Y]$ denotes the bounded linear operators from $X$ to $Y$ where $X$ and $Y$ are Banach spaces. 


$$
\begin{aligned}
& m_{1}^{\prime}=m_{1}=\left(x_{1}, y_{1}\right) \quad \text { where } x_{1} \in l_{2}, y_{1}=\left(y_{1}^{1}, y_{1}^{2}, \ldots\right) \in c_{0} \\
& m_{2}^{\prime}=m_{k_{2}}=\left(x_{k_{2}}, y_{k_{2}}\right) \quad \text { where } \sup _{j \geqq k_{2}}\left|y_{1}^{(j)}\right|<\frac{1}{2} \\
& \vdots \quad \vdots \\
& \begin{array}{l}
m_{j}^{\prime}=m_{k_{j}}=\left(x_{k_{j}}, y_{k_{j}}\right) \quad \text { where } \sup _{j \geqq k_{n}}\left|y_{k_{n}-1}^{(j)}\right|<\frac{1}{2^{n-1}} . \\
\vdots
\end{array} \quad \vdots
\end{aligned}
$$

We now divide each $y_{k_{j}}$ into three parts. We write $y_{k_{j}}=y_{k_{j}}^{+}+y_{k_{j}}^{*}+y_{k_{j}}$, where $P_{k_{j}} m_{k_{j}}=\left(x_{k j}, y_{k_{j}}\right)$. According to (I) $y_{k_{j}}$ has the following properties:

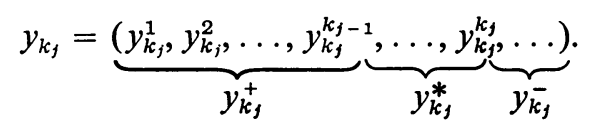

Starting with $j=4$ via (I) and (II), we have $\left\|y_{k,}^{*}\right\| \geqq 3 / 4$ and by (III) for different $j$ the $y_{k j}^{*}$ are disjoint. We now show that, after renumbering, $T:\left(\left(\alpha_{j}\right)\right) \rightarrow \sum_{j=1}^{\infty} \alpha_{j} m_{j}^{\prime}$ is an isomorphism of $c_{0}$ onto a subspace of $M$.

$$
\begin{aligned}
\left\|\sum_{1}^{\infty} \alpha_{j} m_{j}\right\| & =\left\|\sum_{1}^{\infty} \alpha_{j} x_{k_{j}}\right\|_{l_{2}}+\left\|\sum_{1}^{\infty} \alpha_{j} y_{k_{j}}\right\|_{c_{0}} \\
& \leqq\left\|\sum_{1}^{\infty} \alpha_{j} x_{k_{j}}\right\|_{l_{2}}+\left\|\sum_{1}^{\infty} \alpha_{j} y_{k_{j}}^{+}\right\|_{c_{0}}+\left\|\sum_{1}^{\infty} \alpha_{j} y_{k_{j}}^{*}\right\|_{c_{0}}+\left\|\sum_{1}^{\infty} \alpha_{j} y_{k_{j}}\right\|_{c_{0}} \\
& \leqq \sup \left|\alpha_{j}\right|\left(\sum_{1}^{\infty} \frac{1}{2} j+1+\sum_{1}^{\infty} \frac{1}{2} j\right) \leqq 3 \sup \left|\alpha_{j}\right|, \quad 1 \leqq j<\infty,
\end{aligned}
$$

also

$$
\begin{aligned}
\left\|\sum_{1}^{\infty} \alpha_{j} m_{j}\right\| & \geqq\left\|\sum_{1}^{\infty} \alpha_{j} y_{k_{j}}^{*}\right\|_{c_{0}}-\left(\left\|\sum_{1}^{\infty} \alpha_{j} x_{k_{j}}\right\|_{l_{2}}+\left\|\sum_{1}^{\infty} \alpha_{j} y_{k_{j}}^{+}\right\|_{c_{0}}+\left\|\sum_{1}^{\infty} \alpha_{j} y_{k_{j}}^{-}\right\|_{c_{0}}\right) \\
& \geqq \sup \left|\alpha_{j}\right| \cdot \frac{3}{4}-\sup \left|\alpha_{j}\right| \cdot \frac{1}{8}-\sup \left|\alpha_{j}\right| \frac{1}{8} \\
& \geqq \frac{1}{2} \sup \left|\alpha_{j}\right|, \quad 1 \leqq j<\infty
\end{aligned}
$$

where we have used the fact that $\left\{y_{k,}^{*}\right\}$ are disjoint. Combining (IV) and (V) we are done.

2. Characterizations and algebraic properties. Since we have here a generalization of weakly compact operators very much like the generalization of compact operators by strictly singular operators, one might expect a similar characterizing theorem, i.e., $T$ is a.w.c. if and only if for all $M$ it follows that there exists $N \subset M$ such that $\left.T\right|_{N}$ is weakly compact. This is not quite the case.

We will need the following theorem due to Kato and Goldberg [6, III.1.9].

1 Theorem. Let $X$ and $Y$ be Banach spaces and suppose $T \in[X, Y]$ is such that $T$ does not have a bounded inverse on any subspace of finite codimension, then there exists $M \subset X, M$ infinite dimensional and closed such that $T_{M}$ (the restriction of $T$ to $M$ ) is compact. 
2 THEOREM. If $T$ is a.w.c. and $M$ is an infinite dimensional closed subspace of $X$; there is a closed infinite dimensional subspace $N \subset M$ such that $T_{N}$ is weakly compact.

Proof. Case I. $M$ is reflexive.

In this case, every bounded operator on $M$ is weakly compact [6].

Case II. $M$ is not reflexive. Then, no subspace of finite codimension is reflexive. Hence, $T$ does not have a bounded inverse on any such subspace. By Theorem 1 , it follows that there exists $N \subset M$ such that $T_{N}$ is compact, hence, weakly compact.

The reverse implication is not true as is shown in the next example.

3 Definition. A quasi-reflexive space is one where $\operatorname{dim}\left(X^{* *} / J X\right)<\infty$ where $J X$ is the canonical image of $X$ in $X^{* *}$.

4 EXAMPLE. It is shown in [9] that if $M$ is an infinite dimensional subspace of a quasi-reflexive space, then $M \supset N$ infinite dimensional and reflexive. Hence, by taking the identity operator on any such space, we show that a.w.c. operators are not characterized by their restrictions to weakly compact operators.

R. Whitley has suggested the following definition and has proven Theorem 6 independently.

5 Definition. $T \in[X, Y]$ is said to have property $R$ whenever $T$ has a bounded inverse on $M$, infinite dimensional and closed, it follows that $M \supset N$ infinite dimensional and reflexive.

This time we do get a characterization.

6 THEOREM. $T$ has property $R$ if and only if for all infinite dimensional closed $M \subset X$ it follows that there exists $N \subset M, N$ infinite dimensional and closed such that $T_{N}$ is weakly compact.

Proof. Suppose that $T$ has property $R$, but that for every infinite dimensional subspace $N$ of $M, T_{N}$ is not weakly compact. Thus, as in Theorem 2, it follows that there exists $W \subset M$ such that $T_{W}$ is compact; hence, a contradiction.

Now let $T$ have a bounded inverse on $M$ and suppose there exists $N \subset M$ such that $T$ is weakly compact. It is readily seen that $N$ is then reflexive.

7 Remark. Example 4 shows that the class of operators with property $R$ is strictly larger than the class of a.w.c. operators.

8 THEOREM. The class of operators with property $R$ taking $X$ into $X$ forms a closed ideal in $[X] \equiv[X, X]$.

Proof. We first show they form a subspace. Let $M$ be contained in $X$, and let $T$ and $S$ have property $R$. There exists, by Theorem $6, N \subset M, N$ an infinite dimensional closed subspace such that $T_{N}$ is weakly compact. Also, there exists $W \subset N$, $W$ an infinite dimensional closed subspace, such that $S_{W}$ is weakly compact. Since the sum of weakly compact operators is weakly compact [8], $T_{W}+S_{W}$ $=(T+S)_{W}$ is weakly compact and, again by Theorem $6, T+S$ has property $R$.

The remaining properties follow by standard arguments. 
9 THEOREM. The a.w.c. operators taking $X$ into $X$ form a closed subset of $[X]$. Proof. Clear.

We do not know at this time whether the a.w.c. operators form a subspace. However, we do have:

10 TheOREM. Let $T$ be a.w.c. and let $S$ be compact. Then $T+S$ is a.w.c.

Proof. Let $(T+S)_{M}$ have a bounded inverse, $M$ a closed subspace of $X . S$ is compact, by a result of Lacey [6, III.2.3], it follows that there exists $N(\varepsilon) \subset M$ where $N(\varepsilon)$ is of finite codimension in $M$ and $\left\|S_{N}\right\|<\varepsilon$ for any $\varepsilon>0$. If $\|(T+S) m\|$ $\geqq a\|m\|$ choosing $\varepsilon=a / 2$ gives $\|T n\| \geqq\|(T+S) n\|-\|S n\| \geqq a / 2\|n\|$. Hence, $T$ has a bounded inverse on $N$ which is thus reflexive. Since $\operatorname{dim}(M / N)<\infty, M$ is reflexive.

3. When all operators are a.w.c. or have property $R$. In $I .1$ we showed that every nonreflexive subspace of $l_{2} \times c_{0}$ contains a copy of $c_{0}$. An inspection of the proof shows that $l_{2}$ may be replaced by an arbitrary reflexive space $X$.

Hence, we have:

1 THEOREM. If $X$ is reflexive, then every nonreflexive subspace of $X \times c_{0}$ contains a copy of $c_{0}$.

Similar methods give:

2 THEOREM. If $X$ is reflexive, then every nonreflexive subspace of $X \times l_{1}$ contains a copy of $l_{1}$.

As an application of these theorems, we have conditions under which all bounded maps are almost weakly compact.

3 Corollary. Let $Y$ be weakly sequentially complete and $X$ reflexive. Then if $T \in\left[X \times c_{0}, Y\right], T$ is a.w.c.

Proof. If $T$ has a bounded inverse on a nonreflexive subspace, then by Theorem $1, Y$ contains a copy of $c_{0}$.

By [1], $c_{0}$ is not weakly sequentially complete. We have a contradiction since every closed subspace of a weakly sequentially complete space is weakly sequentially complete.

4 COROLlaRY. If $X^{*}$ is a separable conjugate space and $Y$ is reflexive, then all $T \in\left[X^{*}, Y \times c_{0}\right]$ are a.w.c.

Proof. As above, in the contrary case, $X^{*}$ contains a copy of $c_{0}$. By [2, Theorem 4], no separable conjugate space can contain $c_{0}$.

5 COROLlaRY. If $X$ is reflexive and $Y$ has a separable conjugate space, then all $T \in\left[X \times l_{1}, Y\right]$ are a.w.c.

Proof. Suppose not, i.e., $T$ has a bounded inverse on $M$ and $M$ is not reflexive. By Theorem 2, $M$, and hence $T M$, contain a copy of $l_{1}$. We now use the fact that if 
$W \subset Y$ and $Y^{*}$ is separable, then so is $W^{*}$ (if $\left\{y_{n}^{*}\right\}$ dense in $Y^{*}$ consider $\left\{\left.y_{n}^{*}\right|_{W}\right\}$ ). But the conjugate space of $l_{1}$ is $m$ [1], which is not separable.

6 Definition. A Banach space $X$ is said to be somewhat reflexive if every infinite dimensional closed subspace contains an infinite dimensional reflexive subspace [9].

7 Proposition. Let either $X$ or $Y$ be somewhat reflexive, then any $T \in[X, Y]$ has property $R$.

Proof. The conclusion is obvious from the definition of somewhat reflexive spaces.

4. Relation to strictly singular and weakly compact operators. If we restrict our attention to certain spaces we find that the operators above fall into more familiar classes.

1 Remark. Let $X$ be a Banach space with a Schauder basis, $\left\{x_{n}\right\}$ [4]. A sequence $\left\{z_{n}\right\},\left\|z_{n}\right\|=1$ is said to be a block basis with respect to $\left\{x_{n}\right\}$ if $z_{n}=\sum_{\alpha_{n}+1}^{\alpha_{n}+1} a_{i} x_{i}$ where $\alpha_{1}<\alpha_{2}<\cdots$. It is the case [2], that $\left\{z_{n}\right\}$ is a basic sequence if $\left\{x_{n}\right\}$ is.

Two basic sequences $\left\{x_{i}\right\}$ and $\left\{y_{i}\right\}$ are said to be equivalent if $\sum t_{i} x_{i}$ converges if and only if $\sum t_{i} y_{i}$ converges. In this case $\left[x_{i}\right]\left(=\mathrm{sp}\left\{x_{i}\right\}=\right.$ the closed linear span of the $x_{i}$ ) is isomorphic to $\left[y_{i}\right]$ under $A:\left(\sum t_{i} x_{i}\right)=\sum t_{i} y_{i}$ (p. 71 of [5]).

A space is said to be block homogeneous if every block basis is equivalent to the original basis. The spaces $l_{p}, 1 \leqq p<\infty$, and $c_{0}$ are block homogeneous. To see this in $l_{p}$ let $\left\{z_{n}\right\}$ be a block basis, $z_{n}=\sum_{\alpha_{n}+1}^{\alpha_{n}+1} a_{i} x_{i}$, hence,

$$
\begin{aligned}
\left\|\sum_{1}^{N} t_{n} z_{n}\right\|_{l_{p}} & =\left\{\left|t_{1}\right|^{p} \sum_{\alpha_{1}+1}^{\alpha_{2}}\left|a_{i}\right|^{p}+\cdots+\left|t_{N}\right|^{p} \sum_{\alpha_{N}+1}^{\alpha_{N}+1}\left|a_{i}\right|^{p}\right\}^{1 / p} \\
& =\left\{\left|t_{1}\right|^{p}+\cdots+\left|t_{N}\right|^{p}\right\}^{1 / p} \quad \text { since }\left\|z_{n}\right\|=1 .
\end{aligned}
$$

The result is now obvious. It is an open question as to whether or not these are the only block homogeneous spaces.

We will be using the following theorem of Bessaga and Pełczyński [2, Theorem 3].

2 THEOREM. Let $\left\{x_{n}\right\}$ be a basis of $X$. If $\left\{y_{n}\right\} \subset X$ satisfies

(a) inf $\left\|y_{n}\right\|=\varepsilon>0$,

(b) $f_{i}\left(y_{n}\right) \rightarrow 0, \quad i=1,2, \ldots,\left(^{3}\right)$

Then there exists $\left\{y_{n_{k}}\right\}$ which is a basic sequence equivalent to a block basis with respect to the $\left\{x_{n}\right\}$.

It is clear that by using the null spaces of the $\left\{f_{i}\right\}$ that we may, in any infinite dimensional space, find such a sequence [2, C.2].

3 THEOREM. If either $X$ or $Y$ is block homogeneous, then $T$ a.w.c. (T has property $R$ ) implies $T$ is strictly singular or $T$ is weakly compact.

${ }^{(3)}\left\{f_{i}\right\} \subset X^{*}$ is the biorthogonal sequence. If $x=\sum a_{i} x_{i}$ then $a_{i} \equiv f_{i}(x)$ and the $f_{i}$ are continuous [16, p. 207]. 
Proof. If $X$ or $Y$ is reflexive, all bounded maps $T: X \rightarrow Y$ are weakly compact [5]. We may now suppose that $Y$ is block homogeneous and not reflexive. We will show $T$ is strictly singular. To do this, it suffices to show that no infinite dimensional closed subspace, $M$, is reflexive. Suppose to the contrary. By the remark following Theorem 2, $M$ contains a basic sequence $\left\{y_{n_{k}}\right\}$, i.e., $\left[y_{n_{k}}\right] \subset M$ and is thus reflexive. Using the fact that $Y$ is block homogeneous we see that there exist isomorphisms $A, B$, such that $\left[y_{n_{k}}\right] \stackrel{A}{\rightarrow}\left[z_{k}\right] \stackrel{B}{\rightarrow}\left\{x_{k}\right\}$ where $\left\{z_{k}\right\}$ is the equivalent block basis of Theorem 2 and $\left\{x_{k}\right\}$ is the basis of $Y$. Thus $Y$ is reflexive which is a contradiction.

We now consider a somewhat weaker property. Let $X$ be a Banach space, not necessarily with a basis, and suppose that every separable subspace is contained in a subspace isomorphic to a block homogeneous space. We shall denote this property by $\left({ }^{* *}\right)$. It is shown in [7, p. 336] that any separable subspace of $l_{p}(S), 1 \leqq p<\infty$, $S$ an uncountable set, is contained in a block homogeneous subspace isometrically isomorphic to $l_{p}$. The same is true of $c_{0}(S)$.

4 Remark. To show any space $X$ is reflexive, it suffices to show that any separable subspace is reflexive.

5. THEOREM. If $X$ has $\left({ }^{* *}\right)$ and $T$ has property $R$, then $T$ is a.w.c.

Proof. Let $T_{M}$ have a bounded inverse and let $N$ be a separable subspace of $M$, then it suffices by the above remark to show $N$ is reflexive. By hypothesis, $N \subset W$ $\subset X$, where $W$ is isomorphic to $B$ and $B$ is block homogeneous. $T_{N}$ has a bounded inverse so $N \supset Z$ reflexive. Let $A$ be the isomorphism between $W$ and $B$. Then $A(Z)$ is a reflexive subspace of $B$. By the proof of Theorem $3, B$ is then reflexive. Hence, so is $W$ and then so is $N$.

6 Definition. $T \in[X, Y]$ is said to be completely continuous if $T$ maps weakly convergent sequences into norm convergent sequences.

7 THEOREM. If $T$ is a.w.c. or has property $R$, then $T$, completely continuous, implies $T$ is strictly singular.

Proof. Let $T$ have a bounded inverse on $M$, infinite dimensional and closed, and suppose T has property $R$. Then $M \supset N$, infinite dimensional and reflexive, hence, $S(N)$, the unit ball in $N$, is weakly compact and by the Eberlein-Šmuljan theorem weakly sequentially compact. Let $\left\{x_{n}\right\} \subset S(N)$ then $\left\{x_{n_{k}}\right\}$ converges weakly to some $x$. By hypothesis, $T x_{n_{k}} \rightarrow T x$ in norm. Since $T$ has a bounded inverse, $x_{n_{k}} \rightarrow x$ in norm, i.e., $S(N)$ is compact. Hence, $W$ is finite dimensional, a contradiction. Thus $M$ is finite dimensional and $T$ is strictly singular. The obvious modifications yield the proof for the a.w.c. case.

If $\mathrm{CC}$ denotes the completely continuous operators, $K$ the strictly singular, and AW the almost weakly compact. We have shown $\mathrm{AW} \cap \mathrm{CC} \subseteq K$. We do not have equality, for consider the injection $T: l_{p} \rightarrow l_{q}, 1<p<q<\infty$. $T$ is weakly, hence almost weakly compact, and is strictly singular by [7]. But $T$ is obviously not completely continuous.

In [12] the following is proven: 
8 THEOREM. Let $S$ be a compact Hausdorff space and $T: C(S) \rightarrow Z$ then the following are equivalent:

(i) $T$ is strictly singular.

(ii) $T$ does not have a bounded inverse on a subspace is isomorphic to $c_{0}$.

(iii) $T$ is weakly compact.

By c.l.i. we shall mean continuous linear image.

9 Corollary. Let $E$ be c.l.i. of $C(S), S$ a compact Hausdorff space. If $T$ is a.w.c. or has property $R$, then $T$ is weakly compact.

Proof. We have the following

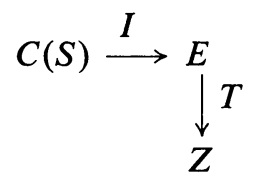

If $T$ is not weakly compact, then since $I$ is onto $T$. $I$ is not weakly compact (if $U$ is the unit ball in $C(S)$, then $I(U)$ is a neighborhood of 0 in $E$ by the open mapping

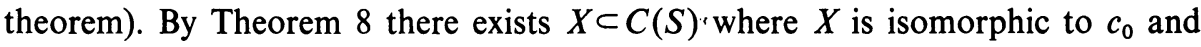
$(T \cdot I)_{X}$ has a bounded inverse. Thus, $I(X)$ is isomorphic to $c_{0}$ and $T$ has a bounded inverse there. The proof is completed by noting that $c_{0}$ is not reflexive nor does it contain any infinite dimensional reflexive subspaces (since then by p. 194 of [1], $c_{0}$ itself would be reflexive).

We note that the theorem is not true for maps into $C(S)$. For let $J$ be the quasireflexive space of R. C. James. $J$ has a basis and is thus separable. Hence, by [1] there exists an isometry, $T: J \rightarrow C[0,1]$. $T$ has property $R$ by I.3.7 but $T$ is not weakly compact since $J$ would then be reflexive.

10 REMARK. If $T$ maps $C(S)$ into $Z$ then, by Theorem $8, T$ a.w.c. or has property $R$ implies $T$ strictly singular.

5. Almost weakly compact compactness and property $R$ under conjugation. We now ask what properties of a.w.c. operators with property $R$ are carried over to their conjugates and vice versa?

In general, it seems that we can say nothing as is seen in the examples below. However, if we restrict our attention to certain types of spaces we do obtain results.

1 EXAmple. Let $T: l_{1} \rightarrow c_{0}$ be an epimorphism, (note: such a $T$ exists by Mazur's theorem [6, II.4.5]. $T$ is a.w.c., since $T$ is strictly singular [7]. But $T^{*}$ is then a monomorphism of $l_{1}$ into $m$. Thus $T^{*}$ is not a.w.c. nor does it have property $R$. $\left(l_{1}\right.$ contains no infinite dimensional reflexive subspaces [1, p. 194].)

2 EXAMPLE. James [10] shows the existence of a space $B$ such that $B^{(\mathrm{iv})}$, the fourth conjugate, is separable and $B^{(\mathrm{iv})}=E \oplus V$ where $E$ is isomorphic to $l_{1}$. In [13] Pełczyński points out that if $T$ is the epimorphism from $l_{1}$ to $B$ ( $B$ is separable) then $T^{* *}$ is strictly singular. Thus, if we let $A=T^{*}$, we see that $A$ is a 
monomorphism of $B^{*}$ (into $m$ ) and $A^{*}=T^{* *}$ is strictly singular. Since $B$ is not reflexive, $A$ is not a.w.c., but $A^{*}$, being strictly singular, is a.w.c.

We leave open the question of the existence of a $T$ such that $T$ does not have property $R$ but $T^{*}$ does.

3 Definition. A Banach space $X$ is said to be subprojective if given $M$, an infinite dimensional subspace of $X$, it follows there exists $N \subset M, N$ infinite dimensional such that $X=N \oplus W[15]$.

4 Theorem. Let $T: X \rightarrow Y$ where $Y$ is subprojective. If $T^{*}$ is a.w.c. then $T$ has property $\mathrm{R}$.

Proof. We proceed as in [15]. Suppose not. Let $T$ have a bounded inverse and suppose $M$ contains no infinite dimensional reflexive subspaces. There exists $W \subset T M$ such that $Y=W \oplus V . W=T N$ for some $N$ contained in $M$. Let $P: Y \rightarrow T N$, then $P^{*}$ takes $(T N)^{*}$ isomorphically into $Y^{*}$. Let $V=P^{*}\left((T N)^{*}\right)$. As in [15], $T_{V}^{*}$ has a bounded inverse, thus $W$ is reflexive.

5 Definition. Let $\left\{x_{n}\right\}$ be a Schauder basis for the Banach space $X$. The basis is said to be unconditional if there exists $\varepsilon>0$ such that $\left\|\sum_{1}^{n} a_{p_{i}} x_{p_{i}}+\sum_{1}^{m} a_{q_{i}} x_{q_{i}}\right\|$ $\geqq \varepsilon\left\|\sum_{1}^{n} a_{p_{i}} x_{p_{i}}\right\|$ for any set of nonoverlapping indices $\left\{p_{i}\right\},\left\{q_{i}\right\}$. See [11] for various equivalences.

Gurevič [8] has shown the following:

6 LEMMA. If $\left\{x_{n}\right\}$ is an unconditional basis for a Banach space $X$, then for $\left\{\alpha_{i}\right\} \in m$ there exists $K, K$ dependent only on $\sup \left|\alpha_{i}\right|$ such that $\left\|\sum_{1}^{n} \alpha_{i} a_{i} x_{i}\right\| \leqq K\left\|\sum_{1}^{n} a_{i} x_{i}\right\|$.

7 Lemma. If $X$ is a Banach space with an unconditional basis $\left\{x_{n}\right\}$, then every nonreflexive subspace of $X$ contains

(i) a complemented copy of $c_{0}$ or

(ii) a complemented copy of $l_{1}$.

Proof. We divide the proof into two cases. Let $M$ be a nonreflexive subspace of $X$.

Case I. $M$ is not weakly sequentially complete. In [3] it is shown that any such subspace contains a copy of $c_{0}$. Now since $X$ is separable, by a result of Sobczyk [14] it follows that this subspace is complemented in $X$.

Case II. $M$ is weakly sequentially complete. Since $M$ is not reflexive, $S(M)$ is not weakly compact. By the Eberlein-S̆muljan theorem, there is a sequence $\left\{y_{j}\right\}$ in the unit ball of $M$ such that $\left\{y_{j}\right\}$ has no weak Cauchy subsequence.

Let $\left\{g_{j}\right\}$ be the coordinate functionals, i.e., if $x \in X$, it follows $x=\sum g_{j}(x) x_{j}$ and suppose $\left\|x_{j}\right\|=1$ for all $j$. By the Cantor process we may extract a subsequence of the $\left\{y_{j}\right\}$ which converges for all $\left\{g_{j}\right\}$. We assume, without loss, that $\left\{y_{j}\right\}$ is the desired subsequence. Since $\left\{y_{j}\right\}$ is not weak Cauchy, there exists $f,\|f\|=1$, and $\varepsilon>0$ such that for all $N$, there exists $n, m>N$ such that

$$
\left|f\left(y_{n}-y_{m}\right)\right|>\varepsilon .
$$


Pick $N$ and then $n_{1}$ and $m_{1}$. Let $z_{1}=y_{n_{1}}-y_{m_{1}}$. Choose $N_{1}$ such that $\left\|\sum_{N_{1}}^{\infty} a_{i}^{(1)} x_{i}\right\|$ $<\varepsilon C / 4$ where $C<1$ and $z_{1}=\sum_{N_{1}}^{\infty} a_{i}^{(1)} x_{i}$. By (I) choose $n_{2}, m_{2}>N_{1}$ such that $\left|f\left(y_{n_{2}}-y_{m_{2}}\right)\right|>\varepsilon$ and $\left\|\sum_{1}^{N 1} a_{i}^{(2)} x_{i}\right\|<\varepsilon C / 4$ where $z_{2}=y_{n_{2}}-y_{m_{2}}=\sum_{1}^{\infty} a_{i}^{(2)} x_{i}$. Note that such a $z_{2}$ may be found since the $\left\{y_{j}\right\}$ converge for each $g_{j}$ and $a_{i}^{(2)}=g_{j}\left(y_{n_{2}}-y_{m_{2}}\right)$. Continuing we get

$$
z_{j}=\sum_{1}^{\infty} a_{i}^{(j)} x_{i}, \quad\left\|\sum_{1}^{N_{j}} a_{i}^{(j+1)} x_{i}\right\|<\frac{\varepsilon}{2^{j+1}} C
$$

and

$$
\left\|\sum_{N j+1} a_{i}^{(j+1)} x_{i}\right\|<\frac{\varepsilon}{2^{j+1}} C
$$

Let

$$
w_{j}=\sum_{N_{j}+1}^{N_{j+1}} a^{(j+1)} x_{i}
$$

Choosing $C$ appropriately, as in [2], we see that $\left\{z_{j}\right\}$ is a basic sequence equivalent to the block basis sequence $\left\{w_{j}\right\}$. We note now, that $C<1,\left|f\left(w_{j}\right)\right|>\varepsilon / 2$. We will now show that $\left[w_{j}\right]$ is isomorphic to $l_{1}$. Since $f$ is of norm $1,2 \geqq\left\|z_{j}\right\| \geqq \varepsilon$. It may then be seen via (II) that $3 \geqq\left\|w_{j}\right\| \geqq \varepsilon / 2$ for all $j$. Therefore, we may assume that $\left\|w_{j}\right\|=1$ and there exists $\delta>0$ such that $\left|f\left(w_{j}\right)\right|>\delta$ for all $j$.

Let $w=\sum a_{i} w_{i} \in\left[w_{j}\right]$ and let $\|w\|=1$. Let $a_{j} f\left(w_{j}\right)=r_{j} e^{-i \theta_{j}}$ applying Lemma 6 we see that tive exists $K$ such that,

$$
\left\|\sum_{1}^{n} a_{j} w_{j}\right\| \geqq K\left\|\sum_{1}^{n} e^{-i \theta_{j}} a_{j} w_{j}\right\| \geqq K\left|f \sum_{1}^{n} e^{-i \theta_{j}} a_{j} w_{j}\right| \geqq K \delta \sum_{1}^{n}\left|a_{j}\right| .
$$

Since this holds for all $n$, we have $\|w\| \geqq K \delta \sum_{1}^{\infty}\left|a_{j}\right|$ also $\|w\| \leqq \sum_{1}^{\infty}\left|a_{j}\right|$. Hence, [ $w_{j}$ ] is isomorphic to $l_{1}$ under $A\left(a_{j}\right)=\sum_{1}^{\infty} a_{j} w_{j}$. Now since $\left[z_{j}\right]$ is equivalent to $\left[w_{j}\right]$ we have shown $M$ contains a copy of $l_{1}$. This result has been shown in a different manner in [3]. Applying C.7 of [2], we obtain the desired result.

Now we can prove

8 THEOREM. If $T: X \rightarrow Y$ where $Y$ is a space with an unconditional basis, then $T^{*}$ a.w.c. implies $T$ is a.w.c.. Further, if the basis is shrinking [4], then $T^{*}$ has property $R$, implies $T$ is a.w.c.

Proof. Suppose not. Then $T$ has a bounded inverse on $M$ where $M$ is not reflexive. Then, by Lemma 7,TM contains $W=T N$ isomorphic to $l_{1}$ or $c_{0}$ and complemented in $Y$. As in Theorem 4, we obtain $V \subset Y^{*}$ such that $T_{V}^{*}$ has a bounded inverse. Here, however, $V$ is isomorphic to $m$ or $l_{1}$, neither of which is reflexive. Hence, if $T^{*}$ is a.w.c., then we have a contradiction. If the basis of $Y$ is shrinking, then $Y$ does not contain any copy of $l_{1}$ [4]. Hence, $V$ is isomorphic to $l_{1}$ which contains no reflexive subspaces. Thus, in this case, it suffices to assume $T^{*}$ has property $R$. 
I wish to express my gratitude to Seymour Goldberg for his constant encouragement and advice. In addition, I would like to thank Robert Whitley with whom I had many stimulating conversations during the course of writing, and A. Pełczyński for comments during his stay at the University of Maryland.

Note added in proof. The problem concerning block homogeneity mentioned at the beginning of paragraph 4 has been solved in the affirmative by M. Zippin, On perfectly homogeneous bases in Banach spaces, Israel J. Math. 4 (1966), 265-272.

\section{BIBLIOGRAPHY}

1. S. Banach, Théorie des opérations linéaires, Chelsea, New York, 1932.

2. C. Bessaga and A. Pełczyński, On bases and unconditional convergence of series in Banach spaces, Studia Math. 17 (1958), 151-164.

3. - A generalization of results of $R$. C. James concerning absolute bases in Banach spaces, Studia Math. 17 (1958), 165-174.

4. M. M. Day, Normed linear spaces, Academic Press, New York, 1962.

5. N. Dunford and J. Schwartz, Linear operators, Part I, Interscience, New York, 1958.

6. S. Goldberg, Unbounded linear operators, McGraw-Hill, New York, 1966.

7. S. Goldberg and E. O. Thorp, On some open questions concerning strictly singular operators, Proc. Amer. Math. Soc. 14 (1963), 334-336.

8. L. A. Gurevič, On unconditional bases, Uspehi Mat. Nauk 8 (1953), no. 5 (57), 153156. (Russian)

9. R. Herman and R. J. Whitley, An example concerning reflexivity, Studia Math. 28 (1967), 289-294.

10. R. C. James, Separable conjugate spaces, Pacific J. Math. 10 (1960), 563-571.

11. C. W. McArthur, A note on subseries convergence, Proc. Amer. Math. Soc. 12 (1961), 540-545.

12. A. Pełczyński, On strictly singular and strictly cosingular operators. I. Strictly singular and strictly cosingular operators in $C(S)$-spaces, Bull. Acad. Polon. Sci. Sér. Sci. Math. Astronom. Phys. 13 (1965), 31-36.

13. - On strictly singular and strictly cosingular operators. II. Strictly singular and strictly cosingular operators in L(v)-spaces, Bull. Acad. Polon. Sci. Sér. Sci. Math. Astronom. Phys. 13 (1965), 37-41.

14. —- Projections in certain Banach spaces, Studia Math. 19 (1960), 209-228.

15. R. J. Whitley, Strictly singular operators and their conjugates, Trans. Amer. Math. Soc. 113 (1964), 252-261.

16. A. Wilansky, Functional analysis, Blaisdell, New York, 1964.

UNIVERSITY OF ROCHESTER, ROCHESTER, NEW YORK 